\title{
Nonlinear Constraints from Non-Abelian Internal Symmetries
}

\author{
Ludwik Turko \\ Institute of Theoretical Physics, University of Wroclaw, \\ pl. Maksa Borna 9, 50-204 Wroctaw, Poland*
}

(Dated: Talk presented at August 29, 2001)

\begin{abstract}
Symmetry invariant local interaction of a many body system leads to global constraints. We obtain explicit forms of the global macroscopic condition assuring that at the microscopic level the evolution respects the overall symmetry.
\end{abstract}

\section{INTRODUCTION}

Let us consider a multiparticle interacting system with the internal symmetry. The problem arises what are global constraints of the system due to the symmetry conservation. In the microscopic formulation with symmetry invariant dynamical equations the answer is given by an analysis of corresponding solutions — assuming that solutions are known.

We are looking here for global conditions to provide consistency with the overall symmetry of the system. These conditions should not depend an exact analytic form of the solutions. An old fashioned example would be Kepler's laws in the classical mechanics which are related to the orbital momentum conservation and can be proved without knowledge of the analytic solutions of Newton equations. As an another very simple example let us consider $N^{(a)}, N^{(b)}, \ldots, N^{(n)}$ charged particles with corresponding individual charges $q_{a}, q_{b}, \ldots, q_{n}$. Particle numbers are time-dependent but the global charges must be conserved (exact $U(1)$ symmetry). So there is a condition

$$
q_{a} \frac{d N^{(a)}}{d t}+q_{b} \frac{d N^{(b)}}{d t}+\cdots+q_{n} \frac{d N^{(n)}}{d t}=0,
$$

valid for any charge conserving interaction.

Our aim 1$]$ is to find a corresponding condition for non-abelian symmetries.

\section{GENERALIZED PROJECTION METHOD}

On the first Bielefeld conference in 1980 I presented a paper [2] devoted to the problem of a formulation of thermodynamics with internal symmetries taken into account. Group projection techniques allowed for a consistent treatment of equilibrium systems and gave tools to obtain canonical partition functions corresponding to the system transforming under given representation of the symmetry group. This technique can also be used for a more general non-static problem.

Let us consider a system consisting of particles belonging to multiplets $\alpha_{j}$ of the symmetry group. Particles from the given multiplet $\alpha_{j}$ are characterized by quantum numbers $\nu_{j}$ - related to the symmetry group, and quantum numbers $\zeta_{j}$ characterizing different multiplets of the same irreducible representation $\alpha_{j}$.

The number of particles of the specie $\left\{\alpha, \nu_{\alpha} ; \zeta\right\}$ is denoted here by $N_{\nu_{\alpha} ;(\zeta)}^{(\alpha)}$. These occupation numbers are time dependent until the system reaches the chemical equilibrium. However the representation of the symmetry group for the system remains constant in the course of a time evolution. A multiplicity $N^{\left(\alpha_{j}\right)}$ of the representation $\alpha_{j}$ in this product is equal to a number of particles which transform under this representation:

$$
N^{\left(\alpha_{j}\right)}=\sum_{j}\left(\sum_{\zeta_{j}} N_{\nu_{\alpha_{j}} ;\left(\zeta_{j}\right)}^{\left(\alpha_{j}\right)}\right)=\sum_{j} N_{\nu_{\alpha_{j}}}^{\left(\alpha_{j}\right)} .
$$

We introduce a state vector $\left|N_{\nu_{\alpha_{1}}}^{\left(\alpha_{1}\right)}, \ldots, N_{\nu_{\alpha_{n}}}^{\left(\alpha_{n}\right)}\right\rangle$ in particle number representation. The probability that $N_{\nu_{\alpha_{1}}}^{\left(\alpha_{1}\right)}, \ldots, N_{\nu_{\alpha_{n}}}^{\left(\alpha_{n}\right)}$ particles transforming under the symmetry group representations $\alpha_{1}, \ldots, \alpha_{n}$ combine into a state

*Electronic address: turko@ift.uni.wroc.p 
transforming under representation $\Lambda$ of the symmetry group is given by

$$
\overline{\left.P_{\left\{N_{\nu_{1}}, \ldots, N_{\nu_{n}}\right.}^{\Lambda, \lambda_{\Lambda}}\right\}}=\left\langle N_{\nu_{\alpha_{1}}}^{\left(\alpha_{1}\right)}, \cdots, N_{\nu_{\alpha_{n}}}^{\left(\alpha_{n}\right)}\left|\mathcal{P}^{\Lambda}\right| N_{\nu_{\alpha_{1}}}^{\left(\alpha_{1}\right)}, \ldots, N_{\nu_{\alpha_{n}}}^{\left(\alpha_{n}\right)}\right\rangle
$$

The projection operator $\mathcal{P}^{\Lambda}$ has the form (see e.g. [3]):

$$
\mathcal{P}^{\Lambda}=d(\Lambda) \int_{G} d \mu(g) \bar{\chi}^{(\Lambda)}(g) U(g) .
$$

Here $\chi^{(\Lambda)}$ is the character of the representation $\Lambda, d(\Lambda)$ is the dimension of the representation, $d \mu(g)$ is the invariant Haar measure on the group, and $U(g)$ is an operator transforming a state under consideration. In particle number representation the operator $U(g)$ is defined as:

$$
\begin{aligned}
& U(g)\left|N_{\nu_{\alpha_{1}}}^{\left(\alpha_{1}\right)}, \ldots, N_{\nu_{\alpha_{n}}}^{\left(\alpha_{n}\right)}\right\rangle \\
& \quad=\sum_{\nu_{1}^{(1)}, \ldots, \nu_{n}^{\left(N_{\nu_{n}}\right)}} D_{\nu_{1}^{(1)} \nu_{1}}^{\left(\alpha_{1}\right)} \cdots D_{\nu_{1}^{\left(N_{\nu_{1}}\right)} \nu_{1}}^{\left(\alpha_{1}\right)} \cdots D_{\nu_{n}^{(1)} \nu_{n}}^{\left(\alpha_{n}\right)} \cdots D_{\nu_{n}^{\left(N_{\nu_{n}}\right)} \nu_{n}}^{\left(\alpha_{n}\right)}\left|N_{\nu_{\alpha_{1}}}^{\left(\alpha_{1}\right)}, \ldots, N_{\nu_{\alpha_{n}}}^{\left(\alpha_{n}\right)}\right\rangle .
\end{aligned}
$$

$D_{\nu, \nu}^{\left(\alpha_{n}\right)}$ is a matrix elements of the group element $g$ corresponding to the representation $\alpha$.

One gets finally

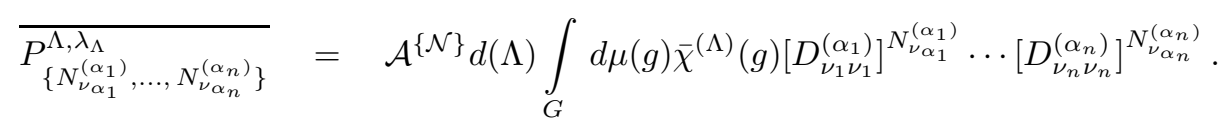

$D_{\nu, \nu}^{\left(\alpha_{n}\right)}$ is a matrix elements of the group element $g$ corresponding to the representation $\alpha$ and $\mathcal{A}^{\{\mathcal{N}\}}$ is an overall permutation normalization factor:

$$
\mathcal{A}^{\{\mathcal{N}\}}=\prod_{j} \prod_{\zeta_{j}} \mathcal{A}_{\left(\zeta_{j}\right)}^{\alpha_{j}}
$$

where $\mathcal{A}_{\left(\zeta_{j}\right)}^{\alpha_{j}}$ are partial factors for particles of the kind $\{\alpha, \zeta\}$ :

$$
\mathcal{A}_{(\zeta)}^{\alpha}=\frac{\mathcal{N}_{(\zeta)}^{(\alpha) !}}{\prod_{\nu_{\alpha}} \mathcal{N}_{\nu_{\alpha} ;(\zeta)}^{(\alpha)} !}
$$

The permutation factor gives a proper normalization of state vectors reflecting indistinguishability of particles:

$$
\left\langle N_{\nu_{\alpha_{1}}}^{\left(\alpha_{1}\right)}, \cdots, N_{\nu_{\alpha_{n}}}^{\left(\alpha_{n}\right)} \mid N_{\nu_{\alpha_{1}}}^{\left(\alpha_{1}\right)}, \ldots, N_{\nu_{\alpha_{n}}}^{\left(\alpha_{n}\right)}\right\rangle=\mathcal{A}^{\{\mathcal{N}\}}
$$

Because of symmetry conservations all weights in Eq. (6) should be constant:

$$
\frac{d}{d t} \overline{\left.P_{\left\{N_{\nu_{1}}\right.}^{\left(, \lambda_{\Lambda}\right)}, \ldots, N_{\nu_{\alpha_{n}}}^{\left(\alpha_{n}\right)}\right\}}=0
$$

Introducing here the result of Eq. (6) one obtains:

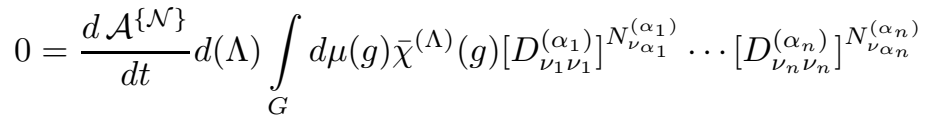

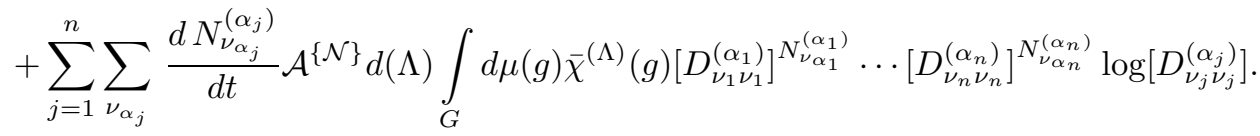

The integrals which appear in Eq. (11) can be expressed explicitly in an analytic form for any compact symmetry group. 
To write an expression for the time derivative of the normalization factor $\mathcal{A}^{\{\mathcal{N}\}}$ we perform analytic continuation from integer to continuous values of variables $N_{\nu_{\alpha_{n}}}^{\left(\alpha_{n}\right)}$. All factorials in Eq. (7) are replaced by the $\Gamma-$ function of corresponding arguments. We encounter here also the digamma function $\psi$ [4]:

$$
\psi(x)=\frac{d \log \Gamma(x)}{d x} .
$$

This allows to write:

$$
\frac{d \mathcal{A}^{\{\mathcal{N}\}}}{d t}=\mathcal{A}^{\{\mathcal{N}\}} \sum_{j} \sum_{\zeta_{j}}\left[\frac{d \mathcal{N}_{\left(\zeta_{j}\right)}^{\left(\alpha_{j}\right)}}{d t} \psi\left(\mathcal{N}_{\left(\zeta_{j}\right)}^{\left(\alpha_{j}\right)}+1\right)-\sum_{\nu_{\alpha_{j}}} \frac{d \mathcal{N}_{\nu_{\alpha_{j}} ;\left(\zeta_{j}\right)}^{\left(\alpha_{j}\right)}}{d t} \psi\left(\mathcal{N}_{\nu_{\alpha} ;\left(\zeta_{j}\right)}^{\left(\alpha_{j}\right)}+1\right)\right]
$$

Eq. [11) can be written in a form

$$
\begin{aligned}
& \sum_{j=1}^{n} \sum_{\nu_{\alpha_{j}}} \frac{d N_{\nu_{\alpha_{j}}}^{\left(\alpha_{j}\right)}}{d t} \frac{d \log \widetilde{\mathcal{P}}_{\left\{N_{\nu_{1}}^{\left(\alpha_{1}\right)}, \ldots, N_{\nu_{n}}^{\left(\alpha_{n}\right)}\right\}}^{\left(\alpha_{\alpha_{j}}\right.}}{d N_{\nu_{\alpha_{j}}}^{\left(\alpha_{j}\right.}} \\
& \quad=\sum_{j} \sum_{\zeta_{j}}\left(-\frac{d N_{\left(\zeta_{j}\right)}^{\left(\alpha_{j}\right)}}{d t} \psi\left(N_{\left(\zeta_{j}\right)}^{\left(\alpha_{j}\right)}+1\right)+\sum_{\nu_{\alpha_{j}}} \frac{d N_{\nu_{\alpha_{j}} ;\left(\zeta_{j}\right)}^{\left(\alpha_{j}\right)}}{d t} \psi\left(N_{\nu_{\alpha_{j}} ;\left(\zeta_{j}\right)}^{\left(\alpha_{j}\right)}+1\right)\right) .
\end{aligned}
$$

where

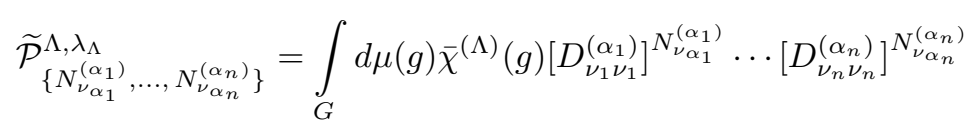

is analytically extended for continuous values of variables $N_{\nu_{\alpha_{j}}}^{\left(\alpha_{j}\right)}$. This gives

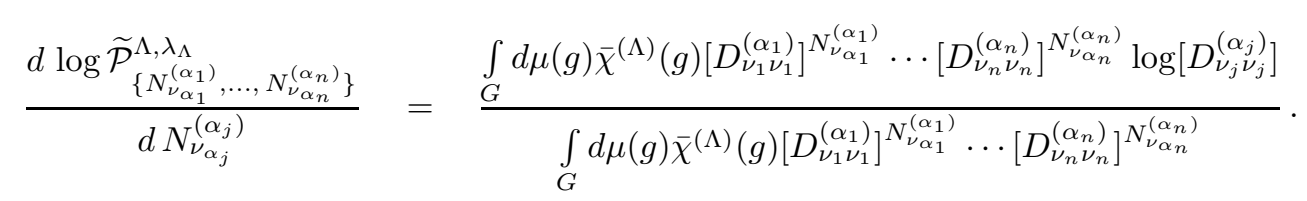

For higher multiplicities digamma functions in Eq. (14) can be replaced by corresponding logarithmic functions according to the asymptotic formula [4]:

$$
\psi(N+1) \approx \log N
$$

Eqs. (14) and (16) give a set of conditions related to the internal symmetry of a system. They are meaningful only for nonzero values of coefficients (6). It is easy to see that coefficients $\overline{P_{\left\{N_{\nu_{1}}, \ldots, N_{\nu_{n}}\right\}}^{\left(\alpha_{1}\right)}}$ are different from zero only if parameters $\lambda_{\Lambda}$ are consistent with the conservation of the simultaneously measurable charges related to the symmetry group. A number of such charges is equal to the rank $k$ of the symmetry group. For the isospin $S U(2)$ group that is the third component of the isospin, for the flavour $S U(3)$ that would be the third component of the isospin and the hypercharge. In general case one has $k$ linear relations between variables $N_{\nu_{\alpha_{j}}}^{\left(\alpha_{j}\right)}$ what reduces correspondingly the number of independent variables.

\section{CONCLUSIONS}

We have got relations which are necessary global conditions to provide consistency with the overall symmetry of the system. They do not depend on the form of the underlying microscopic interaction. Abelian internal symmetries lead to simple and obvious linear relations as in Eq. (1). Non-abelian internal symmetries lead to nonlinear relations as in Eq. (11).

If we know solutions of symmetry invariant evolution equations then all those constraints would become identities. In other case they give a subsidiary information about the system and can be used as a consistency check for approximative calculations. A case of generalized Vlasov - Boltzman kinetic equations was considered in [1]. 
New constraints lead to decreasing number of available states for the system during its evolution. New correlations appear and the change in the thermodynamical behavior can be expected [5].

A challenging point is to find structures which would correspond to chemical potentials when system approaches the equilibrium distribution. The equilibrium distribution in the presence of constraints can be constructed by the Lagrange multipliers method. The multipliers related to the "abelian constraints, such as Eq. (1)), are well known chemical potentials. Multipliers related to the "nonabelian" constraints (11) are more complicated. Because these constraints are nonlinear ones, corresponding multipliers cannot be treated as standard additive thermodynamical potentials.

\section{Acknowledgments}

Work supported in part by the Polish Committee for Scientific Research under contract KBN - 2P03B 03018

[1] L. Turko and J. Rafelski, Eur. Phys. J. C 18 (2001) 587 arXiv:hep-th/0003079].

L. Turko and J. Rafelski, "Macroconstraints from Microsymmetries of Macrosystems," arXiv:nucl-th/0011047.

[2] K. Redlich and L. Turko, Z. Phys. C 5 (1980) 201.

[3] E. P. Wigner, Group Theory and Its Application to the Quantum Mechanics of Atomic Spectra, (Academic Press, New York and London, 1959) .

[4] M. Abramowitz and I. A. Stegun (eds.), Handbook of Mathematical Functions, (National Bureau of Standards, Applied Mathematics Series . 55, 1964).

[5] H. T. Elze, J. Rafelski and L. Turko, Phys. Lett. B 506 (2001) 123 arXiv:hep-ph/0103066. 